\title{
Secondary Reconstruction of Recurrent Primary Intraosseous Meningioma of the Calvarium Using a Fasciocutaneous Anterolateral Thigh Free Flap
}

\author{
Raakhi Mistry ${ }^{1}$, Chris Tsimiklis ${ }^{2}$, Marcus Wagstaff ${ }^{1}$, Amal Abou-Hamden ${ }^{2}$, Yugesh Caplash $^{1}$ \\ ${ }^{1}$ Department of Plastic \& Reconstructive Surgery, Royal Adelaide Hospital, Adelaide, South Australia; ${ }^{2}$ Department of Neurosurgery, \\ Royal Adelaide Hospital, Adelaide, South Australia. \\ Email: raakhimistry@gmail.com
}

Received October $20^{\text {th }}, 2013$; revised November $15^{\text {th }}, 2013$; accepted December $5^{\text {th }}, 2013$

Copyright (C) 2013 Raakhi Mistry et al. This is an open access article distributed under the Creative Commons Attribution License, which permits unrestricted use, distribution, and reproduction in any medium, provided the original work is properly cited.

\begin{abstract}
We present a patient with a primary intraosseous meningioma of the calvarium that recurred three years after initial resection and reconstruction with a free anterolateral thigh (ALT) flap. The patient re-presented with progressive swelling in the same area. Imaging confirmed aggressive tumour recurrence with both intra- and extra-cranial diseases and also extension to the ipsilateral orbit. The original anterolateral free flap was raised on anterior pedicle and further debulking of the tumour was performed. Histology confirmed widely infiltrative atypical meningioma (WHO grade II). This case highlights the benefit of using a fasciocutaneous free flap for reconstruction given the potential for tumour recurrence.
\end{abstract}

Keywords: Primary Intraosseous Meningioma; Fasciocutaneous Free Flap

\section{Introduction}

A 76-year-old man was initially presented with a large mass involving his right frontal scalp which had been growing over a period of five years (Figure 1). The mass was firm and non-tender. He had no lymphadenopathy. He had no significant comorbidities, however, he was reported to have sustained a traumatic injury to his head when he was a teenager. CT scan showed an exophytic lesion measuring $9 \times 5 \times 10 \mathrm{~cm}$ arising from the right frontal bone. The lesion appeared to be composed predominantly of soft tissue with a sunburst pattern of ossification centrally. There was hyperostosis of the frontal bone which extended to involve the roof and lateral wall of the ipsilateral orbit. Post-contrast imaging revealed avid and fairly uniform enhancement. On MRI, dural thickening and enhancement were seen suggestive of dural involvement but there was no convincing evidence of intra-axial disease (Figure 2). Initial differential diagnosis based on radiological features was a malignant process such as osteosarcoma. Staging CT showed no evidence of disease elsewhere.

Pre-operative embolization was performed given the highly vascular nature of the tumour, and the patient subsequently underwent primary resection. The tumour was resected down to the frontal bone and the underlying skull was burred until normal calvarial bone was encountered macroscopically. The temporalis muscle also appeared to be grossly involved and thus was partially excised (Figure 3). A free anterolateral thigh flap was raised to reconstruct the soft tissue defect.

Histology demonstrated a WHO grade I meningioma

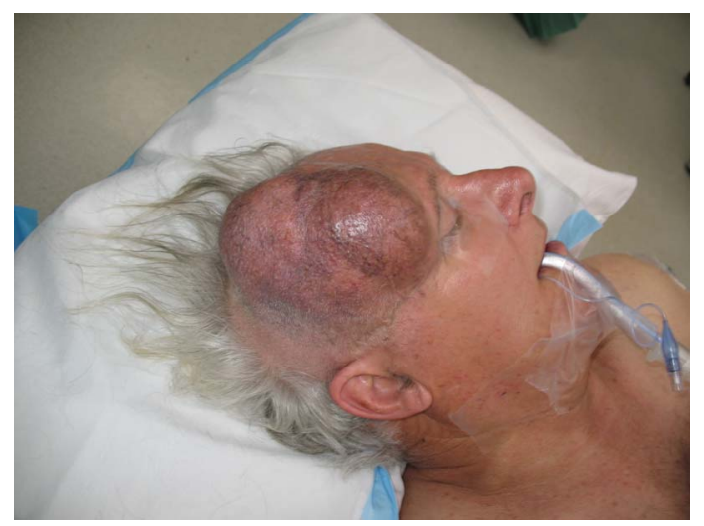

Figure 1. Pre-operative photograph showing the mass on his right frontal scalp on initial presentation. 

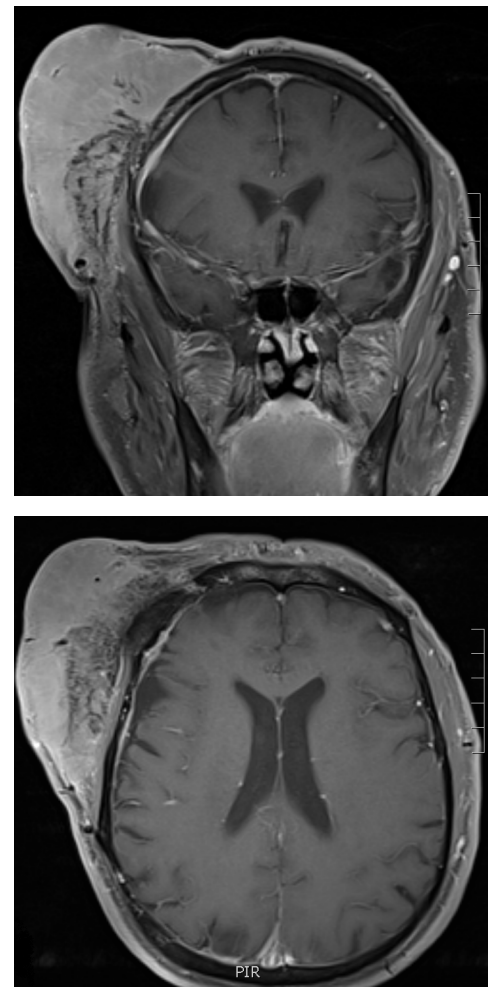

Figure 2. Pre-operative T1 post-Gadolinium coronal (up) and axial (down) slices demonstrating the extent of the tumour and its avid enhancement.

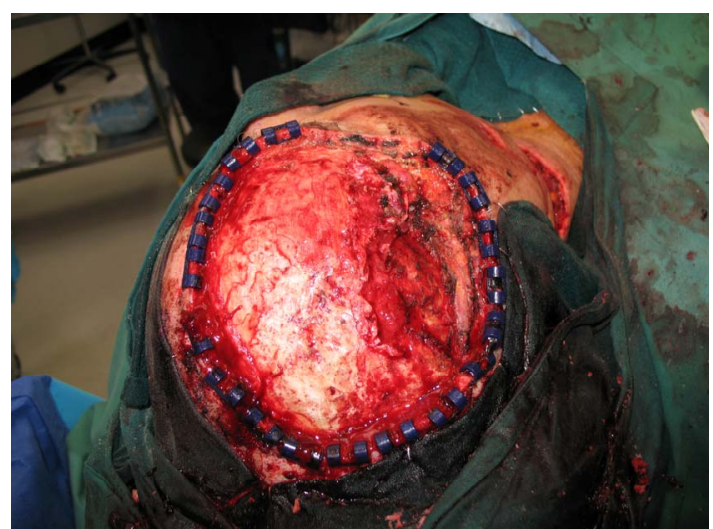

Figure 3. Intra-operative photograph following initial tumour resection.

with some features of atypia. Tumour was present at all of the surgical margins, but the patient elected for surveillance with serial MRI scans rather than for adjuvant therapy.

Three years after his initial surgery, the patient developed swelling under the free flap as well as progressive ptosis of the right upper eyelid. Repeat MRI showed prominent recurrent disease with an intra-cranial component, and extension through the calvarium and diploic space and with a larger extra-cranial soft tissue component extending to involve the superior aspect of the right orbit (Figure 4). Following multidisciplinary discussion, it was felt that the combined treatment with radical surgery and post-operative radiotherapy would improve his quality of life.

Repeat surgery involved carefully raising the flap based on an anterior pedicle to preserve the previously anastomosed vessels. The tumour, which was firmly adherent to the underlying bone and surrounding soft tissue, was debulked. Further resection of the remnant temporalis muscle was necessary. A skull flap was raised and the diseased bone was removed en bloc. The intracranial component of tumour was then resected noting areas of localised pial invasion. The skull defect was reconstructed with titanium mesh. Finally, the orbital roof was raised and the intraorbital component of disease was debulked. This was then reconstructed with titanium plates and screws. The ALT flap was then re-inset. Histology demonstrated an extensively infiltrating atypical meningioma (WHO grade II). The patient made a good postoperative recovery and was subsequently referred to adjuvant radiotherapy (Figure 5).
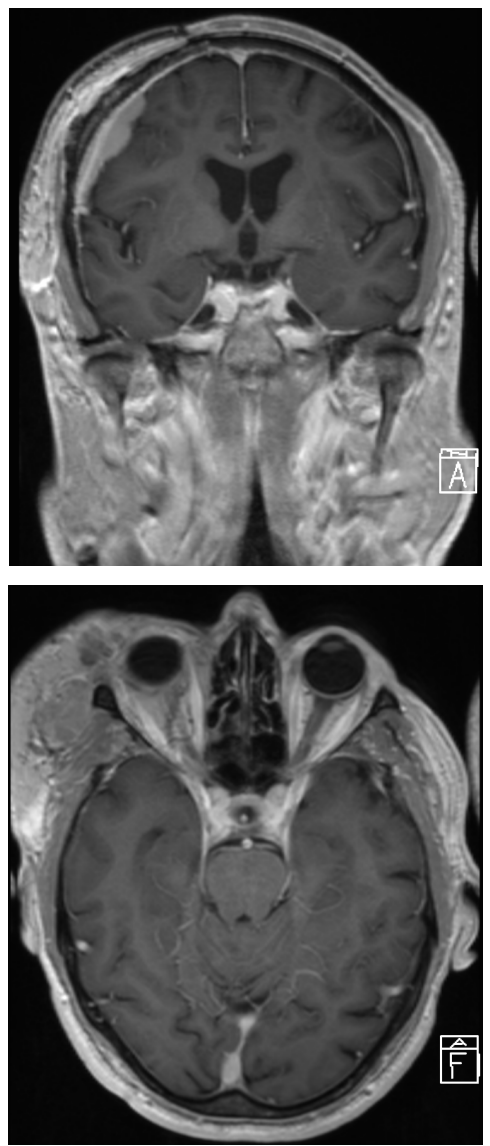

Figure 4. T1 post-gadolinium coronal (up) and axial (down) MRI slices three years after initial resection showing tumour recurrence with involvement of the ipsilateral orbit and extension through the dura. 


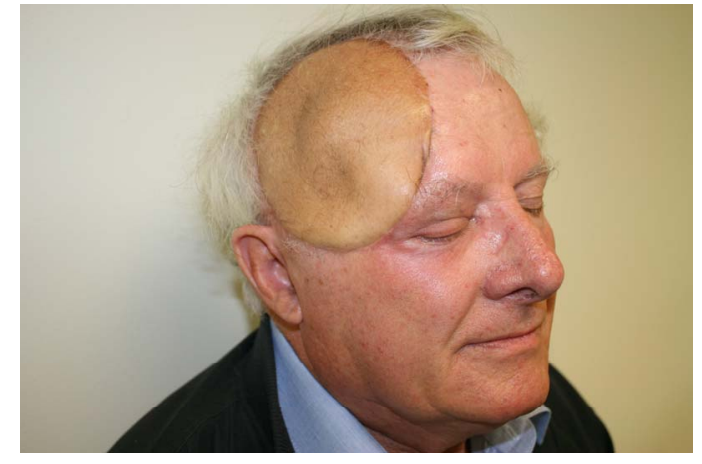

Figure 5. Photograph six weeks post resection of recurrent tumour.

\section{Discussion}

Primary intraosseous meningioma of the calvarium is a subtype of primary extradural meningioma (PEM). In this case, we have demonstrated the potentially aggressive nature of this tumour and possibility for early recurrence.

Meningiomas that arise from locations other than the meninges are known as PEM's [1]. They are a rare type of meningioma, comprising $1 \%-2 \%$ of all meningiomas [2]. A number of extradural sites have been reported in the literature, however the most common is within the calvaria [3]. Several classification systems of PEM's have been described in the literature. Lang et al. recently undertook a retrospective review of PEM's and proposed a classification scheme based on the tumour's relationship to the cranium [3]. Using the schema demonstrated in Table 1 our patient initially had a type IIIC primary intraosseous meningioma.

The aetiology of PEMs is not completely understood but there is evidence to support their development from arachnoid cap cells via several potential mechanisms, including displacement during embryologic development or secondary to a traumatic event [4]. Interestingly, our patient reported having had trauma to the site as a teenager.

In the data reviewed by Lang et al. consisting of a cohort of 142 patients with PEMs, the majority (67\%) of tumours were histologically benign $[3,5]$. Although fewer PEMs were malignant or atypical (33\%), their incidence was higher than observed for intracranial meningiomas. Furthermore recurrence was more likely to occur in type IIB and IIIB tumours compared with type IIC or type IIIC. This may reflect the difficulty in achieving complete macroscopic resection in such cases. The most important factor in prevention of recurrence is the extent of macroscopic resection and the use of the Simpson grading system is commonplace (Table 2) [5]. In our case, a Simpson grade III resection was initially achieved as baseline MRI indicated underlying dural
Table 1. Classification of PEM's by Lang et al. [3].

\begin{tabular}{cl}
\hline Type & \multicolumn{1}{c}{ Tumour relationship to the cranium } \\
\hline I & Purely extracalvarial with no attachment to bone \\
II & Located entirely within the calvarial bone \\
III & Calvarial tumours with extracalvarial extension \\
\hline
\end{tabular}

*Type II and III tumours are then further subdivided as being skull based (B) or convexity $(\mathrm{C})$ lesions depending on their anatomical location.

Table 2. Simpson grading system for surgical excision of meningiomas [5].

\begin{tabular}{cl}
\hline Grade & \multicolumn{1}{c}{ Degree of removal } \\
\hline I $\quad \begin{array}{l}\text { Macroscopically complete removal with excision of dural } \\
\text { attachment and abnormal bone } \\
\text { (including sinus resection when involved) }\end{array}$ \\
II $\quad \begin{array}{l}\text { Macroscopically complete with endothermy coagulation of } \\
\text { dural attachment }\end{array}$ \\
III $\quad \begin{array}{l}\text { Macroscopically complete without resection or coagulation } \\
\text { of dural attachment or of its extradural extensions } \\
\text { (eg. hyperostotic bone) }\end{array}$ \\
IV $\quad \begin{array}{l}\text { Partial removal leaving tumour in situ } \\
\text { V } \quad \text { Simple decompression (+/- biopsy) }\end{array}$ \\
\hline
\end{tabular}

enhancement and thus likely disease involvement.

Other important factors in tumour recurrence are the histological subtype and whether or not adjuvant therapy was undertaken. In one particular study by Barbaro et al., there appeared to be benefit in radiotherapy following partial resection in a series of patients with non-malignant meningiomas [6]. In our case, adjuvant radiotherapy was not undertaken initially given the patient's preference for surveillance.

The ALT free flap is a popular workhorse for reconstruction of head and neck defects. It is based on the lateral circumflex femoral artery and can be raised as a cutaneous, fasciocutaneous or musculocutaneous flap. The advantages of this flap include no need for change in patient position, simultaneous flap harvest, relatively straightforward harvest, adequate pedicle length and calibre and negligible donor site morbidity [7,8]. In this case, a fasciocutaneous ALT free flap was raised and microvascular anastomoses were performed end-to-end to the right superficial temporal vessels. The advantage of using this flap was the ability to raise it on a pedicle and perform further tumour resection when it recurred. Because the flap had well-established vascularity from the wound edges we were able to raise it on an anterior pedicle without threatening its viability. This meant that the patient did not require further soft tissue reconstruction, resulting in faster recovery and sooner commencement of radiotherapy. 
Over the past two decades primary microsurgical reconstruction has become the treatment of choice for head and neck tumours. We are now seeing more patients with recurrent tumours who were initially treated with surgical resection and free flap reconstruction. While the aims of primary reconstruction following tumour resection are to achieve functional and aesthetically acceptable results, it is also important to consider the risk of tumour recurrence when choosing a flap for reconstruction.

\section{Conclusion}

In this case, we have demonstrated a patient with aggressive recurrence of a primary intraosseous meningioma of the calvarium. Although rare, there are increasing reports of such tumours in the literature and they can represent a major challenge in achieving complete resection. When this patient developed tumour recurrence, we were able to raise the original fasciocutaneous flap on a pedicle and perform further resection without threatening the flap's viability.

\section{REFERENCES}

[1] K. I. Desai, T. D. Nadkarni, R. D. Bhayani and A. Goel, "Intradiploic Meningioma of the Orbit: A Case Report," Neurology India, Vol. 52, No. 3, 2004, pp. 380-382.

[2] J. B. Elder, R. Atkinson, C. S. Zee and T. C. Chen, "Primary Intraosseous Meningioma," Neurosurgical Focus, Vol. 23, No. 4, 2007, p. E13. http://dx.doi.org/10.3171/FOC-07/10/E13
[3] F. F. Lang, O. K. Macdonald, G. N. Fuller and F. DeMonte, "Primary Extradural Meningiomas: A Report on Nine Cases and Review of the Literature from the Era of Computerized Tomography Scanning," Journal of Neurosurgery, Vol. 93, No. 6, 2000, pp. 940-950. http://dx.doi.org/10.3171/jns.2000.93.6.0940

[4] E. J. Rushing, J. P. Bouffard, S. McCall, C. Olsen, H. Mena, G. D. Sandberg, et al., "Primary Extracranial Meningiomas: An Analysis of 146 Cases," Head and Neck Pathology, Vol. 3, No. 2, 2009, pp. 116-130. http://dx.doi.org/10.1007/s12105-009-0118-1

[5] D. Simpson, "The Recurrence of Intracranial Meningiomas after Surgical Treatment," Journal of Neurology, Neurosurgery, and Psychiatry, Vol. 20, No. 1, 1957, pp. $22-$ 39. http://dx.doi.org/10.1136/jnnp.20.1.22

[6] N. M. Barbaro, P. H. Gutin, C. B. Wilson, G. E. Sheline, E. B. Boldrey and W. M. Wara, "Radiation Therapy in the Treatment of Partially Resected Meningiomas," Neurosurgery, Vol. 20, No. 4, 1987, pp. 525-528. http://dx.doi.org/10.1227/00006123-198704000-00003

[7] F. C. Wei, V. Jain, N. Celik, H. C. Chen, D. C. Chuang and C. H. Lin, "Have We Found an Ideal Soft-Tissue Flap? An Experience with 672 Anterolateral Thigh Flaps," Plastic and Reconstructive Surgery, Vol. 109, No. 7, 2002, pp. 2219-2226. http://dx.doi.org/10.1097/00006534-200206000-00007

[8] B. S. Lutz, "Aesthetic and Functional Advantages of the Anterolateral Thigh Flap in Reconstruction of Tumor-Related Scalp Defects," Microsurgery, Vol. 22, No. 6, 2002, pp. 258-264. http://dx.doi.org/10.1002/micr.10047 